DESY 95-091

ISSN 0418-9833

ITP-UH-17/95

hep-ph/9505307

May 1995

\title{
HOT SCALAR ELECTRODYNAMICS AS A TOY MODEL FOR HOT QCDE
}

\author{
ULRIKE KRAEMMER, ANTON K. REBHAN \\ DESY, Gruppe Theorie, Notkestr. 85, D-22603 Hamburg, Germany \\ and \\ HERMANN SCHULZ \\ Institut f. Theoret. Physik, Universität Hannover, \\ Appelstr. 2, D-30167 Hannover, Germany
}

\begin{abstract}
Hot scalar electrodynamics is adopted as a toy model for a hot gluon plasma to display some aspects of the compulsory resummation of hard thermal loops when next-to-leading order quantities at soft momentum scales are to be calculated.
\end{abstract}

\section{Introduction}

One of Tanguy Altherr's major activities was the development and application of an improved perturbation theory for quantum field theory at high temperature and/or high density 2. Although his real interest with respect to the former was in hot QCD, which will be hopefully probed more or less directly in heavy-ion collisions in the near future - a future, sadly, without Tanguy — , he naturally was also interested in studying toy models which can give guidance in analysing and overcoming the theoretical problems involved.

A particularly simple toy model is provided by self-interacting scalar fields细, in which some of the issues associated with the resummation of hard thermal loops 5 . can already be elucidated. Here we shall rather take massless scalar fields interacting through electrodynamics to furnish a toy model which is closer to the self-interacting massless gauge bosons of QCD. Notice that spinor QED is a much less interesting toy model since the infrared behaviour of fermions is much milder on account of Pauli suppression. Our toy model Lagrangian thus is

$$
\mathcal{L}=\left(D_{\mu} \phi\right)^{*} D^{\mu} \phi-\frac{1}{4} F_{\mu \nu} F^{\mu \nu}
$$

with $D_{\mu}=\partial_{\mu}+i e A_{\mu}$.

*Talk given by A.K.R. at a one-day meeting dedicated to the memory of Tanguy ALTHERR, held on November 4, 1994 at CERN, Geneva. To appear in a Gedenkschrift published by World Scientific. 
Focussing on the perturbative corrections to the thermal photon self-energy, we shall discuss to what extent the properties of hot gluons and the required resummation methods can be understood already in the Abelian toy model.

\section{Hard thermal loops}

On dimensional grounds, in the limit of high temperature $T$ much larger than any momentum or mass scale, the photon self-energy, as well as the one for the scalar particles, will receive one-loop contributions proportional to $e^{2} T^{2}$. Hence, this will give rise to important modifications of the spectrum on momentum scales $Q_{0}, q \lesssim e T$.

Let us begin with the simpler scalar self-energy. In the imaginary-time formalism, the one-loop self-energy is given by the usual Feynman integrals with the difference that the integral over frequencies is replaced by a sum over imaginary Matsubara frequencies $\omega_{n}=2 \pi n T$,

$$
\Sigma=e^{2} T \sum_{n} \int \frac{d^{3} k}{(2 \pi)^{3}}\left(\frac{3}{K^{2}}-Q^{2} \frac{3-\alpha}{K^{2}(K-Q)^{2}}+\frac{2(\alpha-1) K \cdot Q}{K^{4}(K-Q)^{2}}\right),
$$

where $\alpha$ is the gauge fixing parameter of general covariant gauges. At first, this is defined only for discrete imaginary $Q_{0}$, but can be continued analyticly to real continuous frequencies $Q_{0}$.

In the limit $T \gg Q_{0}, q$, only the first term in (2) turns out to contribute, which happens to be gauge-parameter independent. Evaluating the thermal sum through contour integrals 7 one finds

$$
\begin{aligned}
e^{2} T \sum_{n} \int \frac{d^{3} k}{(2 \pi)^{3}} \frac{3}{K^{2}} & =3 e^{2} T \sum_{n} \int \frac{d^{3} k}{(2 \pi)^{3}} \frac{-1}{(2 \pi n T)^{2}+k^{2}} \\
& =-3 e^{2} \int \frac{d^{3} k}{(2 \pi)^{3}} \frac{n(k)+\frac{1}{2}}{k} \\
& =-\frac{1}{4} e^{2} T^{2} \equiv-\mu^{2}
\end{aligned}
$$

upon dropping an UV-divergent temperature-independent contribution (or using dimensional regularisation), which would be cancelled by the usual zero-temperature mass counterterm.

The result (3) shows that the original massless scalar particles acquire a certain thermal mass. It should be borne in mind, however, that this is not quite the same as a zero-temperature rest mass! For example, calculating the finite-temperature corrections to the energy-momentum tensor, one finds that it remains traceless (up to the usual zero-temperature trace anomaly).

Turning to the less simple photon self-energy, one first of all faces a more complicated tensorial structure because the existence of a preferred frame, the plasma rest-frame, 'breaks' Lorentz symmetry. Actually this means simply that there is an 
additional vector $U_{\mu}\left(=\delta_{\mu}^{0}\right.$ in the plasma rest-frame), to build covariant quantities with. In particular there exists now a vector which is transverse to a given momentum $Q_{\mu}$,

$$
V_{\mu}=Q^{2} U_{\mu}-(U \cdot Q) Q_{\mu},
$$

and this allows one to construct a novel transverse symmetric Lorentz tensor

$$
P_{\ell}=V \otimes V / V^{2}
$$

Together with the usual transverse projector one can build one which is also spatially transverse and orthogonal to $P_{\ell}$,

$$
P_{t}=g-\frac{Q \otimes Q}{Q^{2}}-P_{\ell} .
$$

The photon self-energy $\Pi^{\mu \nu}(Q)$, which is transverse with respect to $Q_{\mu}$, therefore involves two (rather than the usual one) structure functions,

$$
\Pi^{\mu \nu}=\Pi_{t} P_{t}^{\mu \nu}+\Pi_{\ell} P_{\ell}^{\mu \nu}
$$

In the nonabelian case this turns out to be more complicated. Indeed, already the one-loop contribution beyond the leading $T^{2}$ part is nontransverse in gauges other than Feynman 8 . The Abelian case is also special in that the gauge-boson self-energy is manifestly gauge parameter independent. At one-loop order it reads

$$
\Pi^{\mu \nu}(Q)=e^{2} T \sum_{n} \int \frac{d^{3} k}{(2 \pi)^{3}}\left[\frac{(2 K-Q)_{\mu}(2 K-Q)_{\nu}}{K^{2}(K-Q)^{2}}-\frac{2 g_{\mu \nu}}{K^{2}}\right] .
$$

In the limit $T \gg Q_{0}, q$ one obtains

$$
\begin{aligned}
& \Pi_{\ell}=-\frac{Q^{2}}{q^{2}} \Pi_{00}=3 m^{2}\left(1-\frac{Q_{0}^{2}}{q^{2}}\right)\left(1-\frac{Q_{0}}{2 q} \ln \frac{Q_{0}+q}{Q_{0}-q}\right) \\
& \Pi_{t}=\frac{1}{2}\left(3 m^{2}-\Pi_{\ell}\right),
\end{aligned}
$$

with $m=e T / 3$. As concerns this leading temperature correction, the nonabelian gluon self-energy differs only in that one has to replace $e^{2} \rightarrow g^{2}\left(N+N_{f} / 2\right)$ for $\mathrm{SU}(N)$ with $N_{f}$ fermions 10 .

Unlike the scalar self-energy, there is no simple thermal mass term but $\Pi_{t, \ell}$ are nontrivial functions of $Q_{0} / q$. Correspondingly, the poles in the propagator which describe the normal modes of the plasma are not given by a simple mass hyperboloid, but are given by the transcendental equations $Q^{2}=\Pi_{\ell, t}(Q)$. The resulting dispersion laws $Q_{0}=\omega_{\ell, t}(q)$ for the photonic excitations are given in Fig. 1. One finds that the mass $m=e T / 3$ introduced above equals the minimum frequency for which propagating modes $\left(q^{2}>0\right.$, i.e. $q$ real) exist. Whereas the longitudinal branch approaches the light-cone as $q$ is increased, the 'effective mass' of the transverse branch 


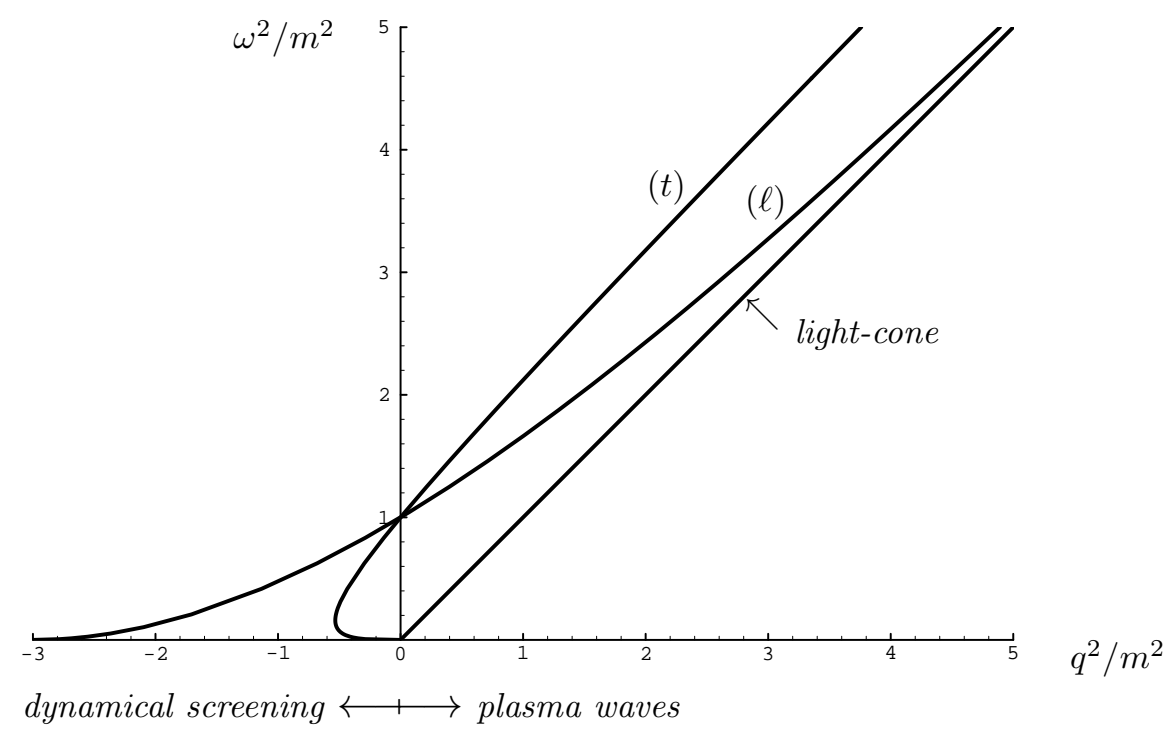

Fig. 1. The dispersion laws of transverse $(t)$ and longitudinal $(\ell)$ photonic (gluonic) quasiparticles

grows with a limiting value $e T / \sqrt{6}$. Also given in Fig. 1 are the poles of the photon propagator for negative $q^{2}$. These do not correspond to normal modes, but describe the response of the system to localised perturbations with given frequency component $\omega<m$. Such perturbations are screened exponentially with screening length $|q(\omega)|$. In the static limit, only the longitudinal branch gives a finite screening length $1 / m_{D}, m_{D}=e T / \sqrt{3}$, which is the familiar Debye screening of electrostatic fields, whereas the transverse branch does not, corresponding to unscreened magnetostatic fields.

In the Abelian case, the absence of a magnetostatic screening mass can be proved to hold exactly 1 , whereas in the nonabelian case, it is known to cause the breakdown of perturbation theory. The common expectation, first put forward by Linde12, is that a magnetic mass is generated nonperturbatively at order $g^{2} T$.

\section{The need for resummation}

Hard thermal loops, the simplest of which is the thermal mass acquired by scalar particles, Eq. (3), typically have integrands which would diverge quadratically in the ultraviolet, if there was no exponential cutoff at the scale $T$ through the distribution function $n$. Hence the term 'hard thermal' - the dominant scale for the loop momentum is $k \sim T$.

The subleading terms in the high-temperature expansion, however, begin to probe smaller momenta, and finally run into infrared problems when bosons are around, for

${ }^{a}$ In renormalizable theories in four dimensions. The hard thermal loops for gravitons 1 , for example, are proportional to $T^{4}$. 
in the infrared, the bosonic distribution function diverges, $n(k) \sim T / k$ for $k \ll T$.

These infrared problems reflect a breakdown of the usual loop expansion. When loop integrals are dominated by the momentum scale $e T$ (or $g T$ in QCD), the thermal masses, which are one-loop objects, become as important as the tree-level kinetic term. Hence, pertuurbation theory breaks down unless these particular one-loop quantities are treated on equal footing with the bare Lagrangian.

In order to rescue perturbation theory one has to resum the hard thermal loops. This can be achieved by adding them to the bare Lagrangian. To avoid overcounting, they have to be subtracted again as 'thermal counterterms', as which they are treated as one-loop objects. This makes sure that the original theory has not changed. This rearrangement of perturbation theory can also be understood as resulting from integrating out in a first step all the hard modes with momentum $k \geq \lambda$, with $\lambda$ such that $g T \ll \lambda \ll T$, which produces an effective Lagrangian containing the hard thermal loops. In a second step, the soft modes with $k \leq \lambda$ are covered, but using the effective Lagrangian.

In general, there are not only thermal masses which are comparable to bare quantities at soft momentum scales, but also hard thermal vertices. In QED and QCD there are in fact infinitely many of those, which have been first classified by Frenkel, Taylor, Braaten, and Pisarski6 0 . The simplicity of scalar field theory is that there are none 1 , which also holds true for scalar electrodynamics14.

The hard thermal self-energies of scalar electrodynamics can be summarized by the following Lagrangian,

$$
\mathcal{L}^{\mathrm{HTL}}=-\mu^{2} \phi^{*} \phi+\frac{3}{4} m^{2} \int \frac{d \Omega}{4 \pi} Y^{\rho} F_{\rho \mu} \frac{1}{(Y \partial)^{2}} F^{\mu \lambda} Y_{\lambda}
$$

where $Y^{\mu}$ is a light-like vector with $Y^{0}=1$. For the scalars, there is just a simple mass term, whereas the photons have a nonlocal effective Lagrangian, corresponding to the more complicated results in Eqs.(9,10). In QED as well as in QCD, similar nonlocalities appear, but because of gauge invariance they involve $[Y \cdot D(A)]^{-1}$, which generates hard thermal vertices with an arbitrary number of external gauge boson lines 15.16 .17$.

\section{4. $\Pi_{\mu \nu}$ beyond leading order}

The leading-order result for the polarization tensor is given by the hard thermal loops (9.10), $\Pi_{\mu \nu} \sim e^{2} T^{2} \sim m^{2}$. The next term in the high-temperature expansion of the bare one-loop result is $\Pi_{\mu \nu}(Q) \sim e^{2} Q T \sim e m^{2}$ for $Q_{0}, q \sim m$, and, were it not for the breakdown of perturbation theory discussed above, two-loop contributions should start with $e^{4} T^{2} \sim e^{2} m^{2}$ and be negligible. However, in the bare theory the $\geq 2$-loop contributions are increasingly infrared-divergent, and also contribute to the relative order $e$ upon resummation. In the following we shall look at the effects of 
this resummation in some simple cases.

\subsection{Static limit of $\Pi_{\ell}-$ electrostatics}

Before resummation, the one-loop result for $\Pi_{\ell}\left(Q_{0}=0, q\right)$ reads

$$
\Pi_{\ell}(0, q)=\Pi_{00}(0, q)=\frac{e^{2} T^{2}}{3}+\frac{e^{2}}{24 \pi^{2}} q^{2} \ln \frac{\sigma}{T}+\ldots=3 m^{2}\left(1+O\left(e^{2}\right)\right)
$$

where $\sigma$ is the renormalization scale, and one would not expect corrections to the

Debye screening mass $m_{D}=\sqrt{3} m$ which are larger than $O\left(e^{2}\right)$. Resumming the thermal mass of the scalars, however, changes the one-loop result to 18

$$
\begin{aligned}
\Pi_{00}(0, q) & =\frac{e^{2} T^{2}}{3}-\frac{e^{2} T}{\pi^{2}} \int_{0}^{\infty} d k\left\{\frac{\mu^{2}}{k^{2}+\mu^{2}}+1-\frac{k}{q} \ln \left|\frac{2 k+q}{2 k-q}\right|\right\}+O\left(e^{2} q^{2} \ln (T)\right) \\
& =\frac{e^{2} T^{2}}{3}-\frac{e^{2} T \mu}{2 \pi}+O\left(e^{2} q^{2} \ln (T)\right) \\
& =3 m^{2}\left(1-\frac{3}{4 \pi} e+O\left(e^{2}\right)\right)
\end{aligned}
$$

This result can in fact be derived in a simplified resummation scheme, which has been put forward by Arnold and Espinosa 19: in the imaginary time formalism it is clear that only the static modes need to be resummed; the nonstatic modes have frequencies $\sim T$, so self-energy corrections $\sim e^{2} T^{2}$ can be treated perturbatively. Keeping only the static modes one sees at once that the result (13) has to be momentum independent at relative order $e$, since only the last term in Eq. (8), which corresponds to the seagull diagram, contributes.

In QCD the self-interacting bosons analogously give rise to relative-order- $g$ corrections, but there $\delta \Pi_{00}(0, q)$ does not happen to be a constant. $\delta \Pi_{00}(0, q)$, and also $\delta \Pi_{00}(0, q \rightarrow 0)$, is gauge dependent, even after resummation 20 , which for some time was taken as indication that the nonabelian Debye mass cannot be extracted from the gluon propagator 2 . The resolution 22 is that $\delta \Pi_{00}(0, q)$ has to be evaluated at the location of the pole in the leading-order gluon propagator, which is at $q= \pm i m_{D}$. There a gauge independent result can be extracted, which however turns out to be logarithmically infrared divergent unless a magnetic screening mass is assumed.

\subsection{Static limit of $\Pi_{t}-$ magnetostatics}

The unresummed one-loop result for $\Pi_{t}\left(Q_{0}=0, q\right)$, which pertains to the magnetostatic sector, reads

$$
\Pi_{t}(0, q)=-\frac{1}{2} \Pi_{i i}(0, q)=\frac{1}{16} e^{2} q T+\frac{e^{2}}{24 \pi^{2}} q^{2} \ln \frac{\sigma}{T}+\ldots=\frac{3}{16} m q e(1+O(e))
$$


Since this vanishes for $q \rightarrow 0$, there appears to be no generation of a magnetic mass, but if Eq. (14) was correct, this would imply a space-like pole in the magnetostatic propagator

$$
\Delta_{t}(0, q)=\frac{-1}{q^{2}-e^{2} q T / 16},
$$

at $q=e^{2} T / 16$. Static magnetic fields would not decay monotoneously but rather oscillate in space.

However, resummation changes all that and instead yields

$$
\Pi_{i i}(0, q)=\frac{e \mu}{2 \pi}\left[2 \mu-\frac{q^{2}+4 \mu^{2}}{q} \arctan \left(\frac{q}{2 \mu}\right)\right]+O\left(e^{2} \mu^{2}\right) .
$$

The result (14) turns out to be correct only in the limit $q \gg \mu \sim e T$, whereas for $q \rightarrow 0$ the true behaviour is $\propto q^{2}$. This can in fact proved to hold in all orders 23 . Thus there is indeed no magnetic screening mass and also no space-like poles in the magnetostatic propagator.

In QCD the unresummed result is similar to (14), but there the resummation of hard thermal loops only modifies the position of the space-like pole, without completely removing it. The latter is sometimes called the Landau ghost of thermal QCD and it is assumed that the nonperturbative generation of a magnetic mass is what will eventually remove it.

\subsection{Long-wave-length limit - plasma frequency}

In the limit $q \rightarrow 0$ with $Q_{0} \neq 0$ the poles in the transverse and in the longitudinal component of the photon propagator coincide and determine the plasma frequency $\omega_{\text {pl. }} \equiv m=e T / 3$, above which there are propagating normal modes of the plasma.

In the unimproved one-loop result the subleading terms are given by

$$
\Pi_{t, \ell}\left(Q_{0}, 0\right)=\frac{e^{2} T^{2}}{9}-\frac{e^{2} T}{12 \pi} i Q_{0}-\frac{e^{2}}{24 \pi^{2}} Q_{0}^{2} \ln \frac{\sigma}{T}+O\left(e^{2} Q_{0}^{2} T^{0}\right)=m^{2}-\frac{e}{4 \pi} i Q_{0} m+O\left(e^{2} m^{2}\right) .
$$

The next-to-leading order term now is purely imaginary, apparently implying a nonzero damping constant

$$
\gamma=-\frac{1}{2 m} \operatorname{Im} \Pi_{t, \ell}\left(Q_{0}=m, 0\right)=\frac{e^{2} T}{24 \pi}=\frac{e}{8 \pi} m
$$

But after resummation, this changes completely:

$$
\Pi_{t}\left(Q_{0}, 0\right)=\Pi_{\ell}\left(Q_{0}, 0\right)=\frac{e^{2} T^{2}}{9}+\frac{e^{2} T}{2 \pi}\left\{-\mu-\frac{4}{3 Q_{0}^{2}}\left(\left[\mu^{2}-\left(Q_{0} / 2\right)^{2}\right]^{\frac{3}{2}}-\mu^{3}\right)\right\} .
$$

Now this is real at $Q_{0}=m$. The unresummed result is seen to be correct only for $Q_{0} \gg m$ and its imaginary part is due to the fact that with $Q_{0} \gg m$ one is above the threshold for pair production of scalar quasi-particles, whereas $m<2 \mu$. 
The analogous calculation in QCD is equally misleading prior to resummation of hard thermal loops. There the bare one-loop damping constant comes out even gauge dependent and negative, which has caused a lot of confusion for quite some time24, and was in fact the driving force for the development of the Braaten-Pisarski resummation scheme.

After resummation, the QCD result does give a nonzero (and positive and gaugeindependent) damping constant 5 , which is in fact due to higher-order Landau damping rather than quasi-particle pair production.

In scalar electrodynamics, the result (19) implies only a correction to the plasma frequency,

$$
m^{2}+\delta m^{2}=\frac{e^{2} T^{2}}{9}\left(1-\frac{8 \sqrt{2}-9}{2 \pi} e\right) \approx \frac{e^{2} T^{2}}{9}(1-0.37 e) .
$$

The corresponding calculation in QCD has been performed in Ref. 26, yielding

$$
\left(\delta m^{2} / m^{2}\right)_{\mathrm{QCD}} \approx-0.18 \sqrt{g^{2} N} .
$$

Let us see how far this latter result can be understood by the above result on scalar electrodynamics. From the leading order terms it is clear that $e^{2}$ corresponds to $g^{2} N$, so we might try to apply (19) by inserting the gluonic plasmon mass in place of the thermal mass of the scalars. This would give $\left(\delta m^{2} / m^{2}\right) \approx-0.028 \sqrt{g^{2} N}$, which is over a factor of 6 short of the actual result. Hence, the correction to the QCD plasma frequency is much larger than what might be expected from just the appearance of thermal masses in the loop integrals.

Another point worth mentioning is that the result (19) is not obtained correctly when only the static modes are resummed. Keeping only the static modes and trying an analytic continuation of the result afterwards gives

$$
\begin{aligned}
\left.\delta \Pi_{t, \ell}\left(Q_{0}, 0\right)\right|_{\text {static contr. }} & =-\frac{1}{3} e^{2} T \int \frac{d^{3} k}{(2 \pi)^{3}}\left\{\frac{4 k^{2}}{\left(k^{2}+\mu^{2}\right)\left(k^{2}+\mu^{2}-Q_{0}^{2}\right)}-\frac{6}{k^{2}+\mu^{2}}\right\} \\
& =-\frac{e^{2} T}{6 \pi}\left\{\frac{2}{Q_{0}}\left[\frac{\mu}{Q_{0}}-\sqrt{\frac{\mu^{2}}{Q_{0}^{2}}-1}\right]\left(Q_{0}^{2}-\mu^{2}\right)+\mu\right\} .
\end{aligned}
$$

which obviously disagrees with (19).

The pitfall is that the separation of the zero modes relies on the imaginary time formalism where either $Q_{0}=0$ or $Q_{0} \propto T$, i.e. hard. Thus, a continuation to soft $Q_{0} \neq 0$ is precluded.

\section{Resummation close to the light-cone}

As a final limiting case we shall consider $Q^{2} \rightarrow 0$, which is relevant for corrections to the longitudinal branch of the dispersion laws for large $q$ (see Fig. 1). 
Close to the light-cone the leading order (hard thermal loop) result diverges logarithmically,

$$
\Pi_{00}^{\mathrm{HTL}} \rightarrow-\frac{3 m^{2}}{2} \ln \frac{q^{2}}{Q^{2}} \quad \text { for } Q^{2} \ll q^{2},
$$

which causes the longitudinal branch to approach the light cone exponentially without ever piercing it. The corresponding residue in the longitudinal photon propagator decays exponentially, too, so that this mode is effectively removed from the spectrum for large momenta.

Calculating the next-to-leading order contribution to $\Pi_{\mu \nu}$ through a resummation of the thermal mass of the scalars (which can in fact be done for general $Q_{0}, q$ in terms of elementary functions 14 - albeit with some tedium), one finds that

$$
\delta \Pi_{00} \rightarrow+e \frac{\mu^{2} q}{\sqrt{Q^{2}}} \text { for } Q^{2} \ll q^{2} .
$$

This result implies that the light-cone is approached even quicker ${ }^{\text {a }}$, but there comes a point where the calculation can no longer be trusted. With $Q^{2} \rightarrow 0, \delta \Pi_{00}$ diverges stronger than $\Pi_{00}^{\mathrm{HTL}}$, so that eventually $\left|\delta \Pi_{00}\right|>\left|\Pi_{00}^{\mathrm{HTL}}\right|$ for any arbitrarily small but nonzero value of $e$ - perturbation theory breaks down again.

The origin of this new desaster is in fact already visible in Eq. (22). There is a logarithmic singularity at the light cone which would be lifted by any finite mass for the hard modes generating the hard thermal loop. In fact, in higher orders hard lines will also have repeated insertions of hard thermal loops. Usually, corrections to the hard lines can be treated perturbatively, but the singular behaviour in the vicinity of the light cone spoils this. In the case of scalar electrodynamics, where the only hard thermal loops are self-energy corrections, it is simple to do a further resummation of the hard thermal loops to be inserted in hard lines. This makes a difference only close to the light cone where $\left|Q^{2}\right| \ll m^{2}$. With massive internal lines, one can indeed put $Q^{2}=0$ and perform a high-temperature expansion which yields a finite result,

$$
\lim _{Q_{0} \rightarrow q} \Pi_{00}^{\text {resum. }}=-\frac{e^{2} T^{2}}{3}\left[\ln \frac{2 T}{\mu}+\frac{1}{2}-\gamma_{E}+\frac{\zeta^{\prime}(2)}{\zeta(2)}\right]-\frac{e^{2} T \mu}{2 \pi q^{2}}+O\left(e^{2} q^{2} T^{0}\right)
$$

with $\gamma_{E}$ being Euler's constant and $\zeta$ the Riemann zeta function.

With Eq. (24), there is now a solution to the equation $Q^{2}=\Pi_{\ell}=-\left(Q^{2} / q^{2}\right) \Pi_{00}$ in the limit $Q^{2} \rightarrow 0$ for a finite value of $q$,

$$
q_{\text {crit. }}^{2} /(e T)^{2}=\frac{1}{3}\left[\ln \frac{4}{e}+\frac{1}{2}-\gamma_{E}+\frac{\zeta^{\prime}(2)}{\zeta(2)}\right]-\frac{e}{4 \pi}=\frac{1}{3} \ln \frac{2.094 \ldots}{e}-\frac{e}{4 \pi} .
$$

Hence, the longitudinal dispersion curve does hit the light cone. In fact, it continues also somewhat to space-like momentum. 
For space-like momentum, there is strong Landau damping from

$$
\Im m \Pi_{00}^{\mathrm{HTL}}=\theta\left(-Q^{2}\right) \frac{3 \pi m^{2} Q_{0}}{2 q},
$$

but the sharp discontinuity at the light-cone is also an artefact. After resummation of the hard lines one finds for $Q^{2} \ll e^{2} q^{2}$

$$
\Im m \Pi_{00}^{\mathrm{resum} .}=\frac{9 \pi e^{2} m^{2} Q_{0}}{16 \pi^{2}\left(q-Q_{0}\right)} \exp \left(-e \sqrt{\frac{q}{8\left(q-Q_{0}\right)}}\right)
$$

so that the imaginary part corresponding to Landau damping starts from zero with all derivatives vanishing. There is thus a finite range in $q$ for which weakly damped plasmons with phase velocity $<1$ exist (the group velocity is $<1$ throughout).

An analogous phenomenon also occurs in hot QCD, which opens the possibility of Cerenkov interaction 27. However, in the case of QCD, the hard thermal vertices also contribute; the corresponding calculation still has to be done28.

Another place where the by now well-established resummation program of Braaten and Pisarski breaks down for similar reasons is in the case of soft real photon produc-

tion 9 . Again, higher-order corrections to the hard internal lines are expected to render the result finite 30 , but a corrected systematic scheme has still to be developed.

In the toy model of scalar electrodynamics, the essentials of these at first unexpected problems and their resolution are already there and give a strong hint how they can be overcome in the case of hot QCD.

\section{References}

[1] T. Altherr, Int. J. Mod. Phys. A8 (1993) 5605.

[2] T. Altherr and U. Kraemmer, Astropart. Phys. 1 (1992) 133.

[3] T. Altherr, Phys. Lett. B 238 (1990) 360;

T. Altherr, T. Grandou, and R. D. Pisarski, Phys. Lett. B 271 (1991) 183.

[4] R. R. Parwani, Phys. Rev. D 45 (1992), 4695.

[5] E. Braaten and R. D. Pisarski, Nucl. Phys. B 337 (1990) 569.

[6] J. Frenkel and J. C. Taylor, Nucl. Phys. B 334 (1990) 199.

[7] J. I. Kapusta, Finite-temperature field theory (Cambridge University Press, Cambridge, 1989).

[8] H.-Th. Elze, U. Heinz, K. Kajantie and T. Toimela, Z. Phys. C 37 (1988) 305.

[9] R. Kobes, G. Kunstatter, and K. W. Mak, Z. Phys. C 45 (1989) 129.

[10] O. K. Kalashnikov and V. V. Klimov, Sov. J. Nucl. Phys. 31 (1980) 699;

H. A. Weldon, Phys. Rev. D 26 (1982) 1394.

[11] E. S. Fradkin, Proc. of the Lebedev Institute 29 (1965) 6. 
[12] A. D. Linde, Phys. Lett. B 96 (1980) 289;

D. J. Gross, R. D. Pisarski and L. G. Yaffe, Rev. Mod. Phys. 53 (1981) 43.

[13] A. Rebhan, Nucl. Phys. B 351 (1991) 706;

J. Frenkel and J. C. Taylor, Z. Phys. C 49 (1991) 515;

F. T. Brandt, J. Frenkel, and J. C. Taylor, Nucl. Phys. B 374 (1992) 169.

[14] U. Kraemmer, A. K.Rebhan, and H. Schulz, Ann. Phys. (NY) 238 (1995) 286.

[15] J. C. Taylor and S. M. Wong, Nucl. Phys. B 346 (1990) 115.

[16] R. D. Pisarski, in From fundamental fields to nuclear phenomena, eds. J. A.

McNeil and C. E. Price (World Scientific Publ. Co., Singapore, 1991).

[17] E. Braaten and R. D. Pisarski, Phys. Rev. D 45 (1992) R1827.

[18] O. K. Kalashnikov and V. V. Klimov, Phys. Lett. B 95 (1980) 423.

[19] P. Arnold and O. Espinosa, Phys. Rev. D 47 (1993) 3546.

[20] T. Toimela, Z. Phys. C 27 (1985) 289.

[21] S. Nadkarni, Phys. Rev. D 33 (1986) 3738.

[22] A. K.Rebhan, Phys. Rev. D 48 (1993) R3967; Nucl. Phys. B 430 (1994) 319.

[23] J.-P. Blaizot, E. Iancu, and R. R. Parwani, preprint Saclay-Y95/034 (1995).

[24] K. Kajantie and J. Kapusta, Ann. Phys. (NY) 160 (1985) 477;

U. Heinz, K. Kajantje and T. Toimela, Ann. Phys. (NY) 176 (1987) 218;

T. H. Hansson and I. Zahed, Nucl. Phys. B 292 (1987) 725;

R. Kobes and G. Kunstatter, Phys. Rev. Lett. 61 (1988) 392;

S. Nadkarni, Phys. Rev. Lett. 61 (1988) 396;

U. Kraemmer, M. Kreuzer and A. Rebhan, Ann. Phys. (NY) 201 (1990) 223.

[25] E. Braaten and R. D. Pisarski, Phys. Rev. D 42 (1990) 2156.

[26] H. Schulz, Nucl. Phys. B 413 (1994) 353.

[27] V. P. Silin and V. N. Ursov, Sov. Phys. - Lebedev Inst. Rep. 5 (1988) 43.

[28] F. Flechsig and A. K.Rebhan, in preparation.

[29] R. Baier, S. Peigné, and D. Schiff, Z. Phys. C 62 (1994) 337;

P. Aurenche, T. Becherrawy, and E. Petitgirard, preprint ENSLAPP-A-452-93 (hep-ph/9403320).

[30] A. Niégawa, preprint OCU-PHYS-153-REV (hep-ph/9408117). 